\section{COOPERACIÓN INVISIBLE. EL PROYECTO MARIA (MÉTODOS DE APOYO Y RECURSOS INTERACTIVOS DE APRENDIZAJE)}

\author{
Ángel Fidalgo Blanco \\ Fátima Azucena Fernández Cabanillas \\ Laboratorio de Innovación en Tecnologías de la Información \\ Departamento de Matemática Aplicada y Métodos Informáticos \\ Universidad Politécnica de Madrid
}

\begin{abstract}
The social networks have turned into a major element of the net in the last years. Through them thousands of people share information with some common interest between them. The root cause of the problem is the difficulty of coordinating these presented resources, a labour that gets complicated when people try to recover all this submited information. From the "Laboratorio de Innovación en Tecnologias de la Información" a software has been developed, after 8 years of work, to classify, organize and put together the resources contributed by the members of a resource oriented network.
\end{abstract}

Since development of this work there has born MARIA, a search engine of formative resources focused to children between 8 and 12 years, composed of the collaboration of adult persons that belong to this social network. Througout this article are detailed the characteristics and utilities of this new search engine, based on a social network where his members provide resources from a supportive and disinterested way, obtaining an easy and effective system to be used by all of them who need it.

KEY WORDS: Social networking; resources; technology; ontology; labels; educational innovation; invisible co-operation.

\section{TIPOS DE REDES SOCIALES Y LA COOPERACIÓN INVISIBLE}

Una red social está formada, básicamente, por personas que hacen pública una información con objeto de compartirla con un grupo de personas con las que tiene algún interés común.

Las redes sociales más conocidas como Tuenti, Facebook, LinKedln o Xing son redes orientadas a las personas,

\section{INVISIBLE COOPERATION THE MARIA PROYECT (METHODS OF SUPPORT AND INTERACTIVE LEARNING RESOURCES)}

RESUMEN: Las redes sociales se han convertido en el elemento estrella de la red en los últimos años. A través de ellas miles de personas comparten informaciones con otros con los que tienen algún interés común. El problema actual de algunas de ellas radica en la dificultad a la hora de coordinar esos recursos aportados, una labor que se complica a la hora de intentar recuperar todos esos datos vertidos. Desde el Laboratorio de Innovación en Tecnologías de la Información se ha desarrollado, tras ocho años de trabajo, un software para clasificar, organizar y poner en común los recursos aportados por los miembros de una red orientada al recurso.

Como desarrollo de este trabajo ha nacido MARIA, un buscador de recursos formativos orientados a niños con edades comprendidas entre 8 y 12 años, nutrido de la colaboración de personas adultas que conforman esa red social. A lo largo de este artículo se detallan las caracteristicas y utilidades de este nuevo buscador, basado en una red social en la que sus miembros aportan recursos de un modo solidario y desinteresado, consiguiendo un sistema fácil y eficaz para ser utilizado por todos aquellos que lo necesiten.

PALABRAS CLAVE: red social, recursos, tecnología, ontologias, etiquetas, innovación educativa, cooperación invisible.

es decir, la información que comparten es relativa a las personas que conforman la red (perfiles, fotos, aficiones, amistades, mensajes, comentarios,...). En estas redes si una persona abandona la red, se va con toda la información que ha compartido.

Hay otro tipo de redes sociales, que están más orientadas a los recursos que aportan las personas. Estos recursos no suelen ser información sobre la propia persona. Las redes más conocidas de esta categoría son Youtube, Flickr 
e incluso Wikipedia. En todas ellas lo que priman son los recursos aportados, si una persona abandona la red sus recursos aportados continúan en ella. A su vez, este tipo de redes se dividen en dos: las que tienen crecimiento coordinado y las que lo tienen descoordinado.

Un buen ejemplo de red social orientada al recurso con crecimiento coordinado es la Wikipedia. Son muchas las personas que aportan recursos, pero estos están unidos por un hilo conductor, que suele ser un temario, la relación entre los artículos, enlaces, etc.

Las redes sociales orientadas con crecimiento no coordinado se basan en que los miembros de la red aportan recursos, pero sin ninguna unión aparente entre ellos, ejemplos de este tipo de redes es Youtube o Flickr. En estas redes sus miembros se limitan a subir y compartir recursos, el título y las categorias son los únicos medios para tratar de clasificar dichos recursos.

De forma muy genérica la propia red Internet la podriamos considerar como una red social orientada a los recursos. Se comparten millones de recursos entre los millones de usuarios. Todos sabemos que el principal problema es el crecimiento no coordinado de esos recursos, con lo que se hacen bastante ineficaces las búsquedas generales. Cualquier búsqueda en Internet nos garantiza decenas de miles de recursos, pero la dificultad es, en un tiempo razonable, encontrar el que nos sea más útil.

Una característica común a todos los tipos de redes es que no hay ningún tipo de compromiso ni planificación por parte de sus miembros sobre los recursos a aportar. Por tanto, la clave es cómo coordinar los recursos que no se sabe cuáles son, cuándo se van a aportar y cómo son. A esta forma de cooperar, clasificar y organizar los recursos que aportan las personas la hemos denominado Cooperación Invisible.

Así pues, la cooperación invisible se refiere a la participación de las personas que conforman una red social orientada al recurso. Lo que se ve son los recursos que han aportado las personas pero no las personas que los han aportado. La cooperación invisible se suele producir cuando hay necesidad de los recursos de la red social, por ejemplo, si una persona necesita los recursos de la red durante unos días, su cooperación se limitará a esos días; sin embargo, si hay un usuario que los utiliza habitualmente, su participación será más continua.

EI LITI' comenzó hace ocho años a desarrollar un software de cooperación invisible que permitiese clasificar, organizar y poner en común los recursos aportados por los miembros de una red orientada al recurso con crecimiento no coordinado. Dicho software es hoy en día un producto que se ha aplicado a numerosos proyectos de investigación, desarrollo e innovación y, por supuesto, a la innovación educativa.

\section{EL PROYECTO MARIA}

En este artículo se presentará una red social orientada al recurso, cuyo objetivo es ayudar a niños de edades comprendidas entre 8 y 12 años en sus tareas docentes. La red social está formada por personas adultas que comparten recursos con los que ayudar en las tareas formativas a los niños, para que les resulte más fácil y eficaz.

A través de las caracteristicas de la red social se describirá la forma de interactuar entre las personas, así como el funcionamiento del software para clasificar, organizar y poner en común los recursos aportados; todo ello para que los recursos de esa red social puedan ser utilizados de forma eficaz.

\subsection{Componentes de nuestra red social}

Conceptualmente hablando, una red social la podríamos considerar dividida en tres módulos:

1. Las personas.

2. Los recursos que comparten.

3. La tecnología en la que se soporta.

\subsubsection{Las personas}

Es el componente principal de la red social, sin embargo, la clave no está en las personas que configuran la red social, sino en la cooperación entre las mismas. La cooperación puede ser muy básica, como por ejemplo poner unas fotos para que el resto de la red las vea y comente si lo desea, o compleja como construir un portal sobre la minería romana. 
Las personas de una red social son como el chasis de un coche, y el motor del mismo sería la cooperación. Ambos, chasis y motor, configuran el coche, por tanto, en una red social "viva" debe haber personas y debe haber cooperación.

En diferentes etapas del proyecto MARIA, han participado personas con distintas tipologías y roles:

- En una primera etapa fueron alumnos del curso de doctorado "Gestión del conocimiento en las organizaciones" que, como caso práctico, adaptaron el software para que personas adultas aportasen recursos útiles.

- En una segunda etapa, fueron más de un centenar de personas (principalmente madres y padres que ayudaban a sus hijos en tareas formativas). Hay que destacar que, inicialmente, no se sabía ni qué recursos iban a aportar ni en qué tareas formativas se iban a utilizar (aunque sí se tenía claro que no iban a ser las tareas que ya hacian en el colegio).

- En una tercera etapa, se sumaron alumnos de primeros cursos de distintas titulaciones universitarias, quienes aportaron recursos más especializados y a menudo desconocidos por las madres y padres que estaban utilizando la red.

- En estos momentos, y coincidiendo con la publicación de este artículo, la red social se abre a todo el público, para que se puedan beneficiar de los recursos aportados por los miembros de la red.

\subsubsection{Los recursos}

Es la información que aportan las personas que configuran la red social. En las redes sociales orientadas a las personas, los recursos suelen ser información sobre las propias personas; el principal problema es que cuando estas personas dejan la red social se llevan los recursos.

En las redes sociales orientadas a los recursos, éstos suelen ser relativos a una temática concreta $y$, si las personas abandonan la red, estos recursos suelen quedar.

Para cualquier red es interesante que los recursos compartidos queden dentro de la misma, además, si éstos están organizados de tal forma que facilite su uso por parte de la red mucho mejor.
Habitualmente, en este tipo de redes los recursos que se "suben" son propios; por ejemplo sus fotos, videos que han grabado, comentarios, etc.

En este caso los recursos no son propios, son recursos que existen en Internet, de acceso público y que se pueden utilizar. Lo que hacían los miembros de la red es comprobar que un determinado recurso era útil para determinadas actividades. Es decir, se establece una relación entre recurso útil, actividad, perfil (niños de 8 a 12 años) y contexto (el hogar). Para conocer si un recurso es útil debemos conocer la actividad en la que lo vamos a utilizar. Como ya se ha comentado anteriormente, el principal problema de este proyecto es que a priori no se sabian las actividades que se iban a realizar con los niños.

En el estudio de la gestión de conocimiento se dice que un tipo de conocimiento es saber qué recursos externos (conocimiento creado fuera de nuestra organización) son útiles para mejorar la competitividad de nuestra organización. Ésta era la idea principal.

Aunque posteriormente, se describirá cómo se determinaron las actividades y los recursos útiles, en este apartado se adelantarán los datos.

Las actividades más habituales que las madres y padres hacian en los hogares con sus niños fueron:

\section{- Explicar conceptos difíciles de entender para niños} de 8 a 12 años. Por ejemplo, el concepto de distancia (a partir de 100 metros) es difícil de entender para un niño de 8 años. Entiende los centímetros, un metro, dos, tres $y$, en general, la distancia que él puede "tocar". Sin embargo, distancias como 100 metros, $400 \mathrm{~m}$, kilómetros, etc., no lo entienden muy bien. Un miembro de la red aportó un recurso muy interesante para que entender ese concepto. Se trata del visor SIGPAC ${ }^{2}$, con este visor puedes situar la imagen en la casa del niño, marcar el recorrido que hace, por ejemplo hasta el colegio y así ver la distancia. La idea es decir al niño, "ves desde casa hasta el colegio has recorrido $400 \mathrm{~m}$ ", de esta forma asocia el concepto de distancia a algo habitual. EI SIGPAC tiene todo el territorio español, por tanto se puede aplicar en cualquier situación.

ARBOR CLXXXV EXTRA 2009 139-153 ISSN: 0210-1963 


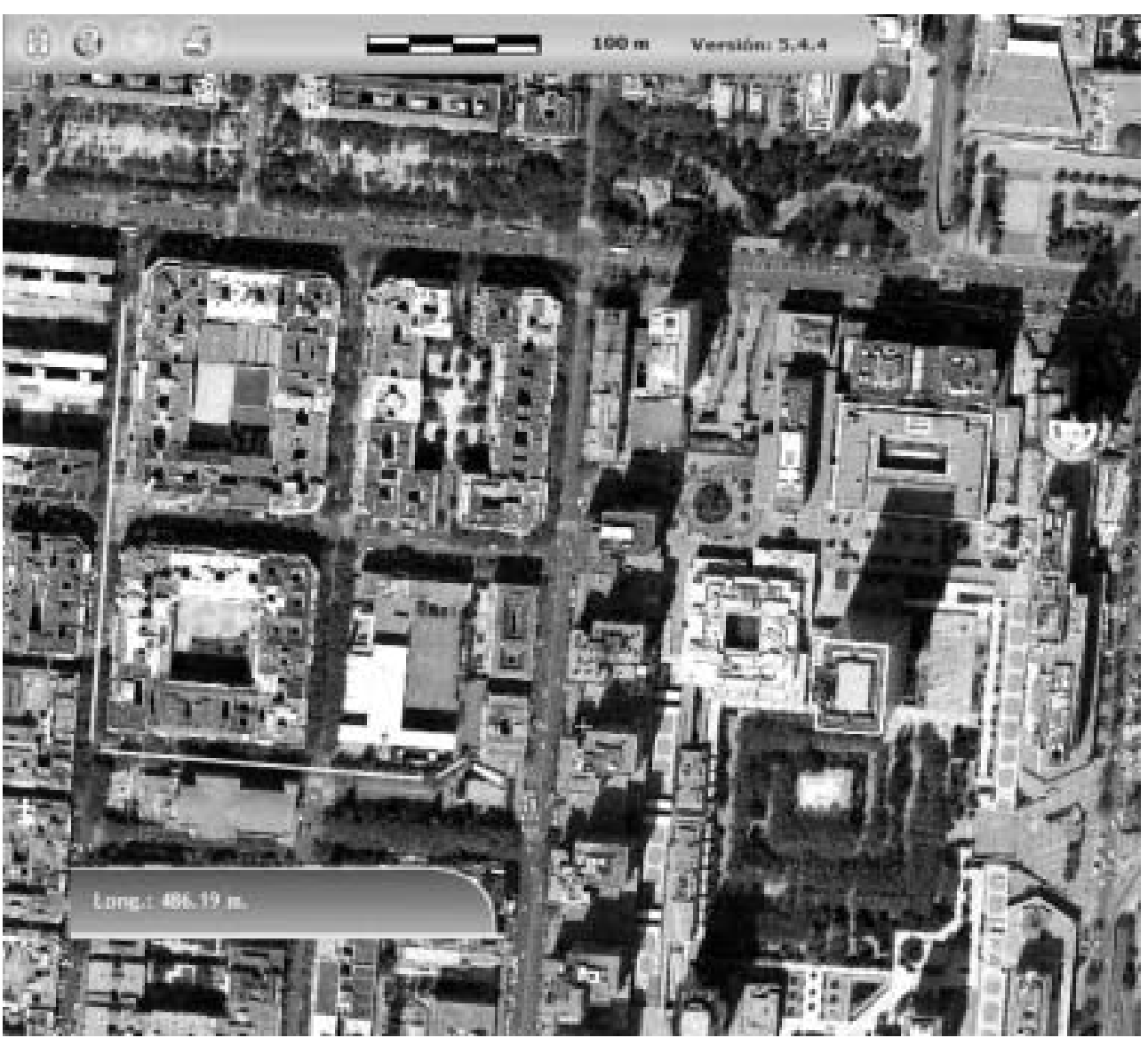

Observe cómo puede trazar la ruta y el sistema indica la distancia. El SIGPAC es un recurso útil para enseñar a un niño de 8 años el concepto de distancia, aunque no se haya diseñado para este fin.

- Ejercicios. Según los miembros de la red, una actividad muy habitual era que el niño hiciese actividades prácticas, para ver que había comprendido un determinado concepto o habilidad (por ejemplo hacer sumas).

- Actividades externas. Se trata de actividades que los niños puedan realizar fuera del ordenador, por ejemplo actividades de comprensión lectora. La idea es que el niño desarrollase destrezas manuales y cognitivas pero con otros medios distintos al ordenador.

Se han resumido los tres tipos de actividades más habituales que hacen las madres y padres con sus hijos, por tanto, ¿qué tipo de recursos tiene la red social MARIA?
Recursos que cumplen tres condiciones:

1. Que son útiles en la realización de las actividades: explicar conceptos difíciles, ejercicios o actividades externas.

2. Que son adecuados para utilizarlos con niños de entre 8 y 12 años.

3. Que se corresponden con el temario de segundo ciclo de primaria.

La mayoría de los recursos encontrados están realizados por profesorado de primaria, aunque otros muchos (como el SIGPAC) están realizados para ser utilizados en otros contextos. Así pues, el conocimiento que los miembros de 
la red han aportando y compartido ha consistido en identificar recursos externos útiles para la misión de la red.

Hay cientos de recursos, pero para que otras personas los puedan utilizar de forma eficaz hay que clasificarlos y organizarlos. Esta importante actividad la han realizado las personas al introducir sus recursos y su propia tecnología.

\subsubsection{La tecnología}

Cualquier software sobre redes sociales se basa en Internet. Es evidente, ya que Internet hoy en día es el canal más eficaz para compartir y visualizar información. Sin embargo, el software que gestiona tanto a las personas como a los recursos que éstas aportan no sirve para cualquier tipo de red; no es lo mismo la gestión que necesita una red orientada a las personas que una red orientada a los recursos.

Las redes sociales orientadas a los recursos más importantes, como youtube o flickr, utilizan los buscadores como principal medio de búsqueda de los recursos compartidos. También los suelen clasificar por temáticas muy generales o por nubes de etiquetas (aunque no suelen ser muy eficaces, ya que las etiquetas, al igual que los recursos, las aportan los usuarios y por tanto hay demasiada información).

Sin embargo, en las redes sociales donde la idea es compartir recursos y organizarlos de forma coordinada, el software existente no es muy adecuado, ya que el crecimiento suele ser divergente, en lugar de convergente.

Los tres módulos son importantes, es como un trípode, basta con que falte un solo apoyo para que se tambalee y caiga al suelo. Las tres "patas" son importantes, no podríamos decir cual es más pero esa no es la cuestión, la cuestión es que las tres son necesarias para sostener el tripode.

La tecnología desarrollada por el LITI permite organizar los recursos, aún cuando no se sabe ni qué recursos van a contener ni para qué se van a utilizar. Este software se denomina $\mathrm{COCO}^{3}$, y actualmente es bastante utilizado en proyectos y convenios con entidades tanto públicas como privadas

\section{El MÉTOdO UTILIZAdO PARA ORGANIZAR LA RED SOCIAL}

Este ejemplo real servirá para entender cómo funciona la tecnología. Para ello debemos situarnos en el momento que el primer usuario encontró un recurso útil. El control de calidad de este recurso es muy sencillo: si sirve para realizar la actividad con el niño es útil, en caso contrario no lo sería.

Cuando el primer usuario encontró el recurso útil, por ejemplo el SIGPAC, sabía que era para una

1. Actividad: explicar un concepto difícil de entender para el niño.

2. Materia con la que estaba trabajando, Tema: Matemáticas.

3. Apartado: distancia.

La ventaja de utilizar el recurso es que sabes dónde lo has utilizado (Matemáticas-distancia) y para qué (para que el niño entienda un concepto difícil). Todo esto es la información que sube a la red social. El primer miembro de la red es quién toma la decisión de crear esa información. Hay dos tipos de ontologias: La fuerte y la débil.

La ontología fuerte es el formulario que se emplea para introducir el recurso, en este caso está compuesto por los campos de: título, descripción, fecha, web, fuente, fichero y sugerencia ${ }^{4}$.

Las ontologías débiles las definen y organizan los propios usuarios. El sistema COCO permite definir dos tipos: categorías y etiquetas. Un conjunto de etiquetas define una categoría. A su vez, tanto las categorías como las etiquetas pueden guardar entre sí una relación jerárquica.

En el ejemplo el primer usuario definió las ontologías: Acción didáctica (categoría) y dentro de ella la etiqueta "Explicación", de la misma forma definió la categoría "Formato del Recurso" y la etiqueta "Interactivo".

Observe que para la categoría "Temario" eligió las etiquetas "Matemáticas" y "La medida de Longitud", pero estas dos últimas guardan una relación jerárquica.

El siguiente usuario que introduce otro recurso sigue el mismo proceso. Si el recurso se corresponde con las eti- 
LABDRATORID DE INANGVACIÓN EN

\begin{tabular}{|c|c|}
\hline Recursos didactioos & \\
\hline That: & 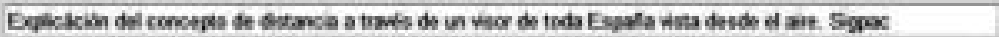 \\
\hline Descripcinin: & 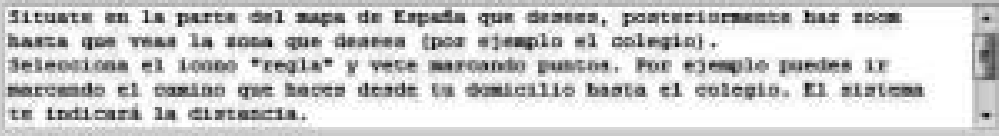 \\
\hline Fachar & t912025 \\
\hline Derecoitn Wetr & 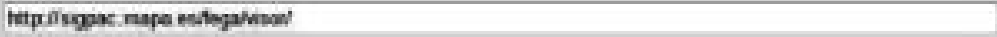 \\
\hline Funste: & Mrintería de Medo Ambiarte \\
\hline Fictere & Eanda: \\
\hline & 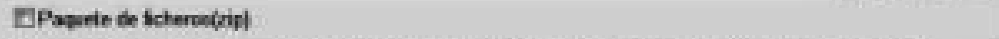 \\
\hline (10) Eeflcasin & \\
\hline FOMMATO DEL MECUNSO & \\
\hline [9 interactive & \\
\hline TENavio & \\
\hline 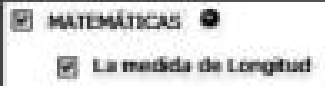 & \\
\hline
\end{tabular}

ACCION DOÁCTCA

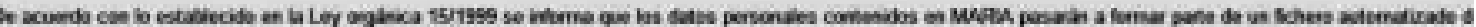

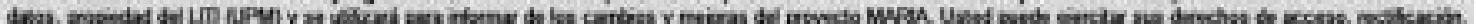

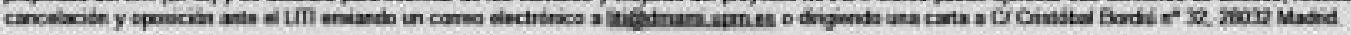

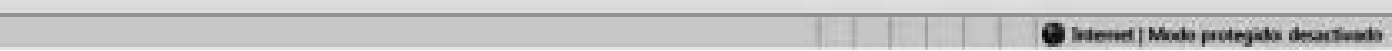

En esta figura se puede observar una ontología fuerte (el formulario para introducir el recurso didáctico) y ontologías débiles (creadas por el primer usuario que introduce el primer recurso útil).

quetas que ha creado el primer usuario las marca, en caso contrario crea otras nuevas, pero siguiendo siempre las mismas categorías; es decir, puede crear las etiquetas que desee pero respetando las categorias (aunque si lo considera necesario podría crear nuevas categorías).
Todo el proceso se repite una y otra vez, al cabo de unos meses y después de haber participado más de 100 usuarios que utilizaron recursos con sus hijos y los subieron a la red, el conjunto de ontologías débiles (etiquetas) creadas fueron las siguientes: 
Para la categoría ACCIÓN DIDÁCTICA

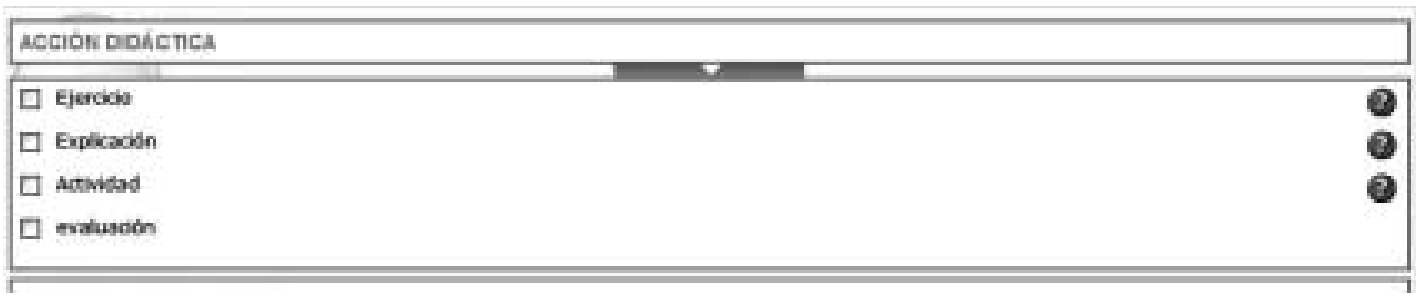

Actualmente hay 201 recursos utilizados para la actividad Explicación, 137 para Ejercicios, 35 para Actividad Externa y 1 para Evaluación. Todo esto significa que la actividad que más realizan en casa las madres y padres con sus niños es explicar conceptos difíciles de entender, seguido de ejercicios.

Para la Categoría: FORMATO

\begin{tabular}{|lr|}
\hline FOHMATO DEL. MECURSO & \\
\hline$\square$ Arimaoin & 0 \\
$\square$ imprimele & 0 \\
$\square$ interactivo & 0 \\
\hline
\end{tabular}

Vemos que los recursos que han identificado como útiles son de tres tipos: interactivos y simulaciones e imprimibles (que se pueden imprimir para posteriormente trabajar con ellos).

Para la Categoría: TEMARIO

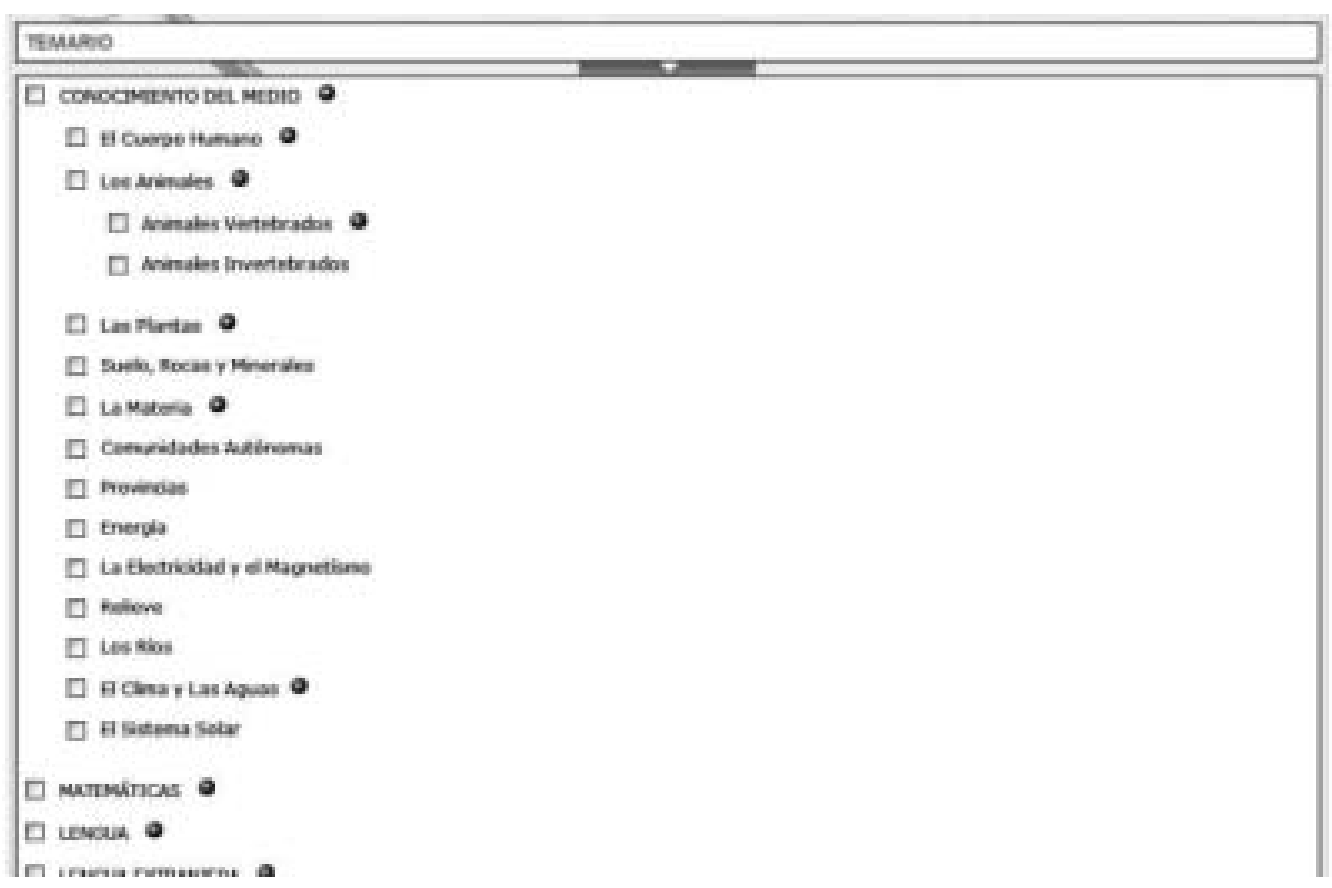


Observe que el temario está formado por un conjunto de etiquetas jerarquizadas, toda esta jerarquía la han construido los propios usuarios. Así pues, el resultado es similar a un índice y en cada apartado hay recursos.

Recuérdese que el índice se ha construido a partir de los recursos que se han ido introduciendo, no al revés.

A través de este sencillo método no sólo clasificamos los recursos, sino que nos permite realizar verdaderos y sistemáticos estudios sobre qué tipo de actividades realizan los padres en sus casas, qué tipo de recursos son más necesarios, en qué materias, etc.

\section{LA EFICACIA DE LA RED SOCIAL}

Cualquier red social se basa en que los constructores son beneficiarios de la misma. Además si la red está bien planteada y con recursos realmente útiles, también pueden servir a personas que, aun sin estar en la red social, tienen el mismo perfil; es decir, que la red social MARIA tendría que ser útil tanto para los miembros de la red social, como para otras madres y padres que tengan niños entre 8 y 12 años, así como para profesorado.

Una vez que la red social tiene recursos útiles y validados (recuérdese que inicialmente la validación la hacía el propio miembro de la red social, al comprobar que el recurso funcionaba para la actividad deseada) se pasa a la segunda fase, que consiste en que el centro de recursos generado por la red social sea útil y eficaz para sus usuarios.

En las redes sociales orientadas al recurso la clave está en encontrar el recurso útil para realizar una determinada actividad. La mayoría de los buscadores realizan la búsqueda a partir de un texto que describe algunas palabras que contiene el recurso, dicho de otra forma, tienes que saber qué recurso quieres utilizar. Sin embargo, en el sistema COCO puedes buscar recursos conociendo la actividad en la que lo quieres utilizar (por ejemplo para explicar un concepto difícil) o en el lugar donde lo quieres utilizar (por ejemplo en el tema de "distancias").

Ésta es la principal diferencia de nuestro sistema con otros buscadores, que puedes utilizar búsquedas por contenidos, por categorias y por etiquetas (bien de forma individual o bien de forma integrada). Esta variedad de búsquedas hace que la red social se transforme en un centro de recurso extremadamente útil y muy eficaz en determinadas situaciones. A continuación se citan algunos ejemplos.

\section{Ejemplo 1. Queremos preparar a un niño en un tema concreto. Búsquedas por "dónde o para qué quiero utilizar el recurso".}

Lo que queremos es que el sistema nos indique qué recursos tiene y para qué sirven respecto a un determinado tema. Esta situación es equivalente a suministrar al buscador el "dónde", en lugar del qué.

En el ejemplo vamos a buscar qué recursos tiene y para qué sirven respecto al tema "Multiplicación y División".

La búsqueda se realizaria marcando directamente el tema (véase primera figura de la página siguiente).

Ahora las etiquetas que sirvieron para clasificar el recurso se utilizan para realizar la búsqueda. Se ha especificado el lugar concreto donde se quiere utilizar los recursos. El resultado de la búsqueda es el que figura en la segunda imagen de la página siguiente.

El centro de recursos de la red social nos indica que hay:

- 4 recursos sobre "Multiplicación y División" que se pueden utilizar para explicar el concepto.

- 16 para adquirir las habilidades de multiplicación y división.

- 5 para realizar actividades externas (fuera del ordenador) sobre multiplicaciones y divisiones.

Este tipo de búsqueda sirve, por ejemplo:

- Para que un profesor o adulto prepare el tema de "Multiplicación y División".

- Para preparar un plan de actividades (se verá posteriormente cómo se hace) sobre el tema concreto.

- Para saber lo que tiene el sistema sobre un tema concreto. Esta acción se recomendaba a todos los miembros de la red antes de subir un recurso concreto, de esta forma se ve si el recurso ya está, o si el que hay es mejor y por tanto no merece la pena subirlo, o al contrario, para ver si el recurso a subir es mejor que el existente y por tanto sí merece la pena subirlo. 


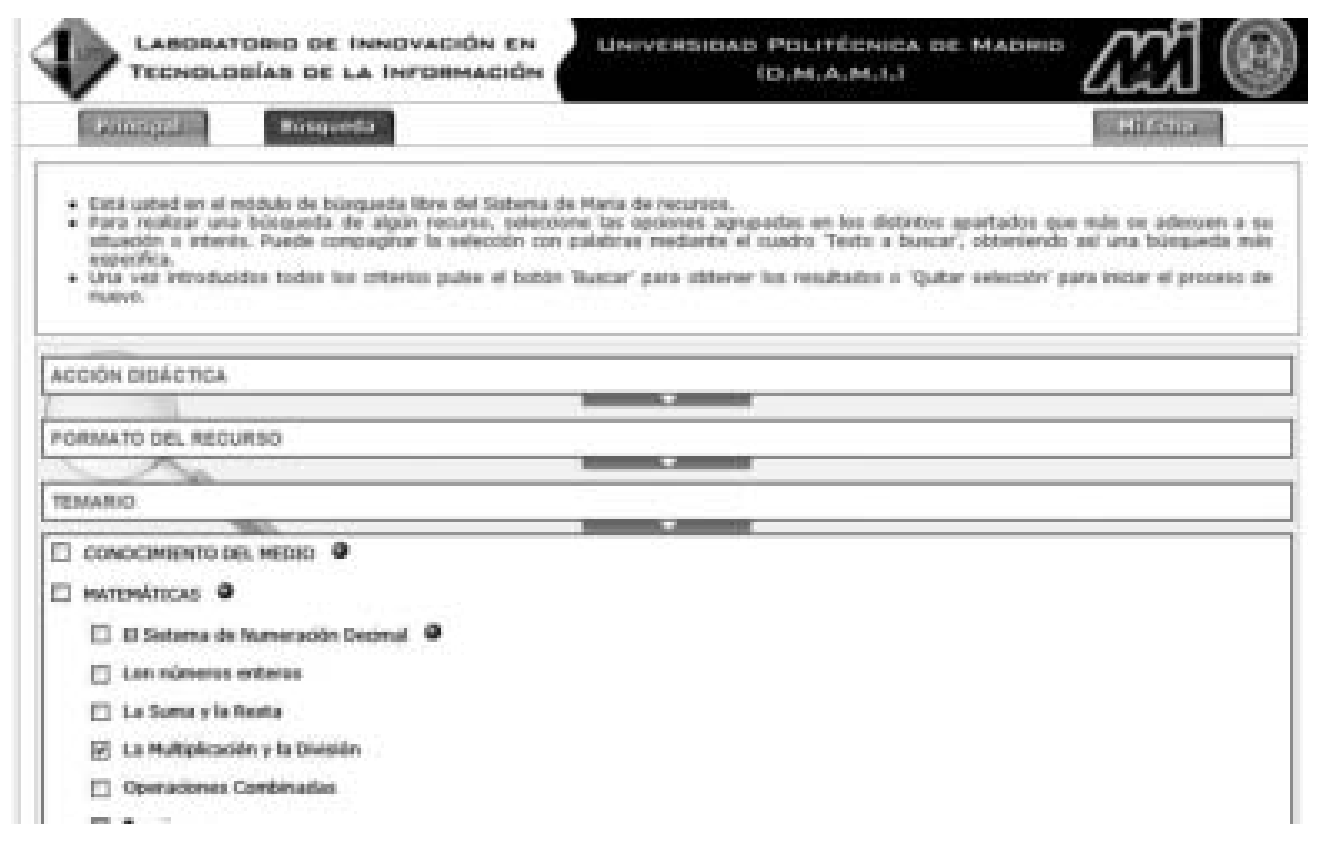

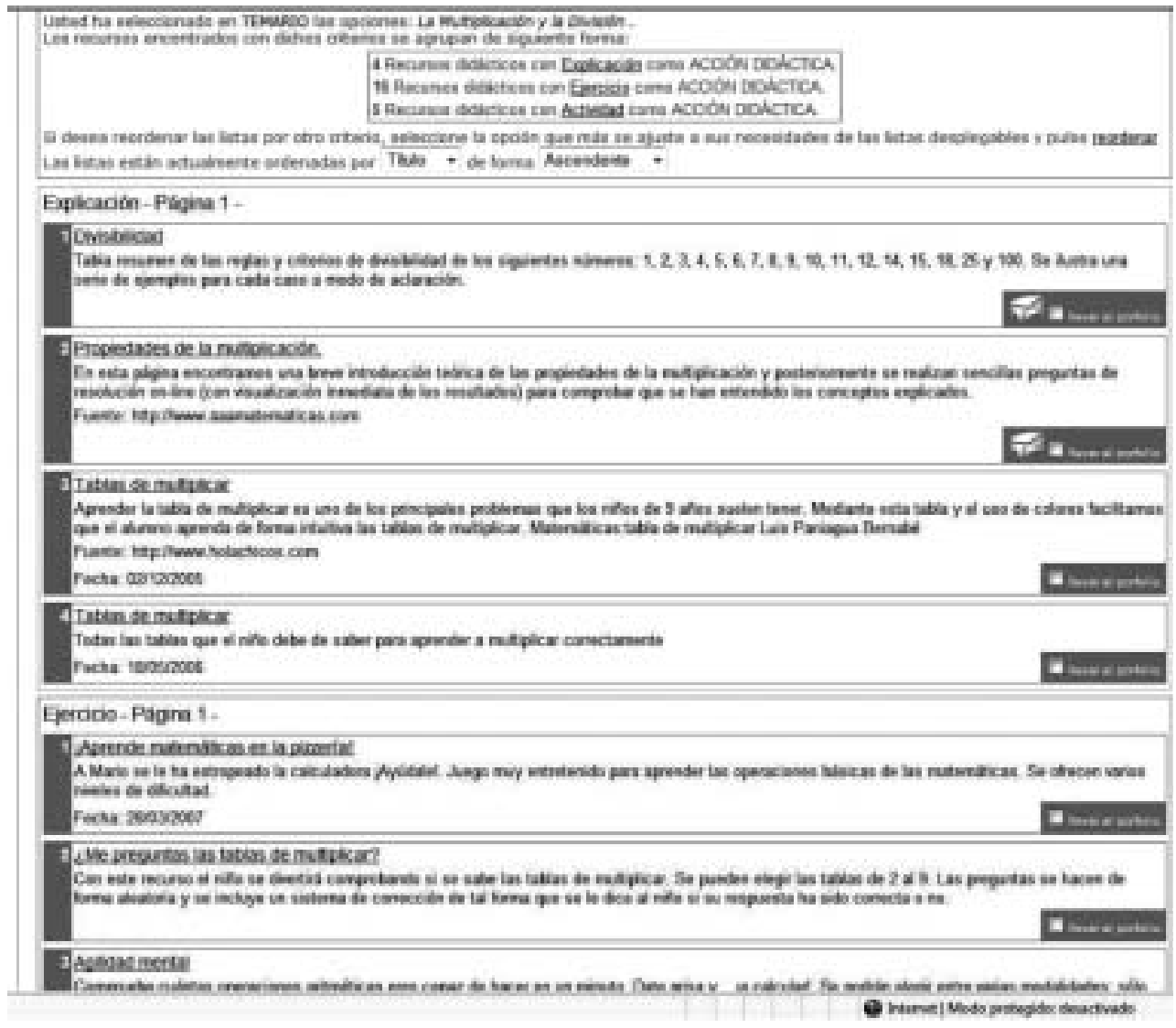


De la misma forma se podría utilizar la búsqueda por etiquetas de una sola categoría. De esta forma si se marca la etiqueta "Explicar" de la categoría "Acción didáctica" tendríamos una búsqueda de un recurso indicando "para qué voy a utilizar el recurso". El proceso de búsqueda sería muy similar al descrito anteriormente.
Ejemplo 2. Búsquedas combinadas. Dónde y para qué quiero utilizar el recurso.

Las búsquedas se pueden utilizar de forma combinada, por ejemplo, si se marca la acción didáctica "Explicar" y el tema "el Sistema Solar"

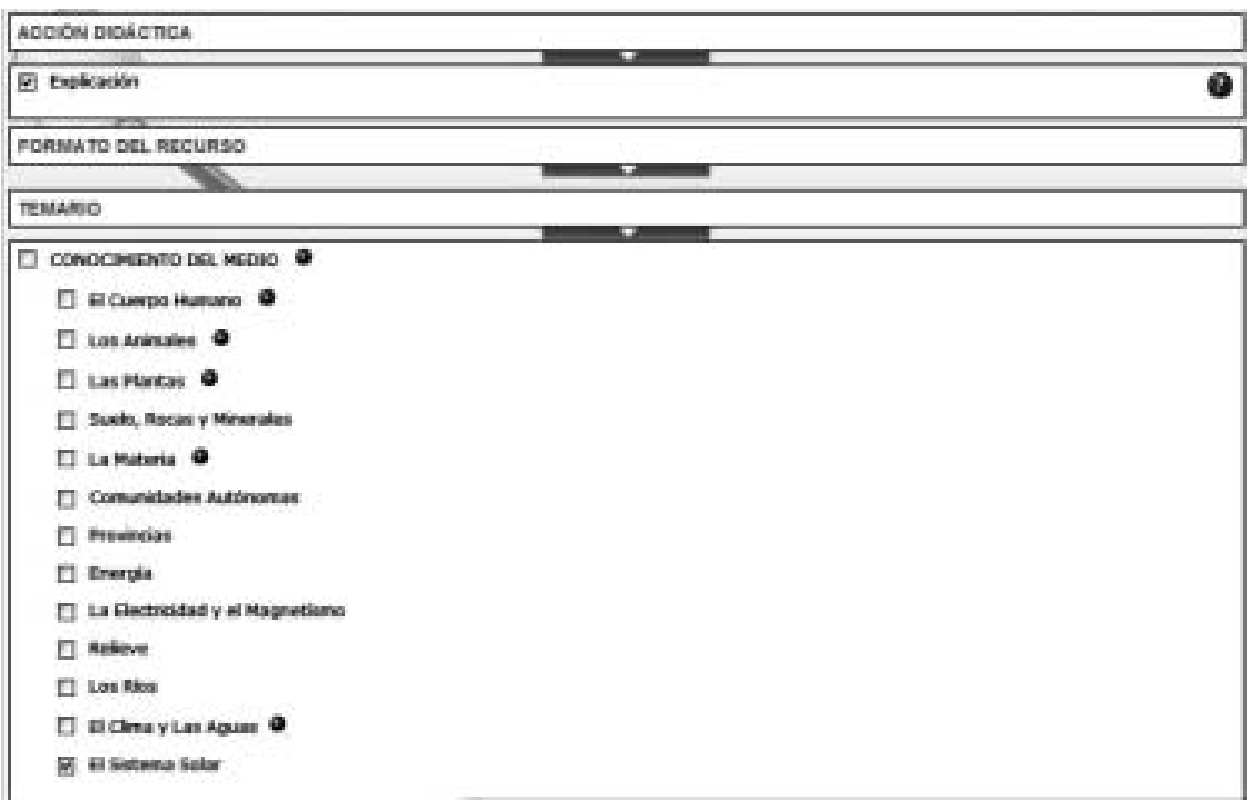

El resultado de esta búsqueda se muestra en la siguiente figura

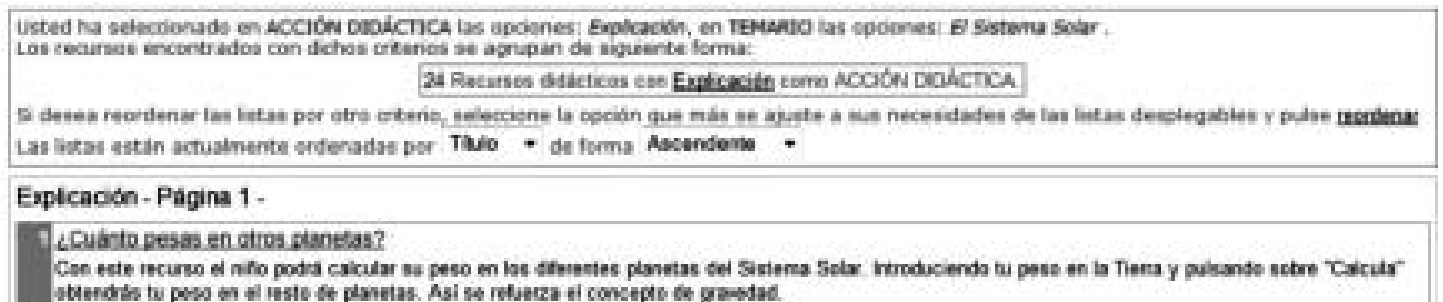

Observe que el sistema ha encontrado 24 recursos sobre el sistema solar que nos sirven para explicar. En este caso significa que los miembros de la red social deberían haber continuado añadiendo nuevas etiquetas que colgaran de "Sistema Solar".
La otra opción es dar más precisión a la búsqueda. Por ejemplo, si se querían recursos para explicar concretamente la rotación, a las etiquetas marcadas se añadirían las de texto. 


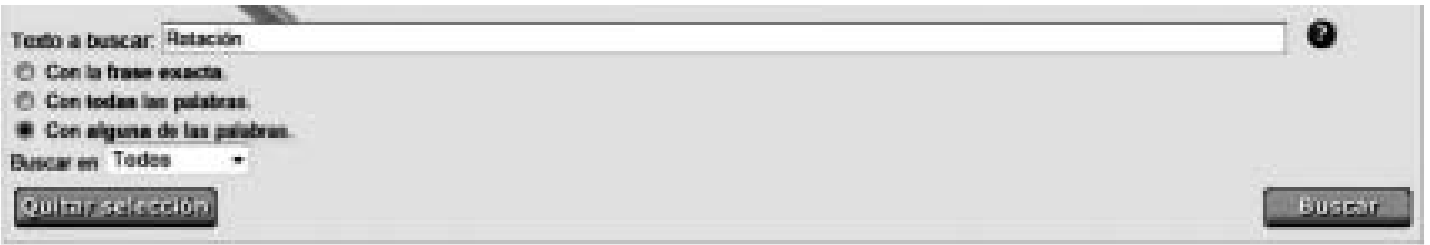

La combinación de búsquedas por categorias, etiquetas jerárquicas y texto darían la máxima precisión. De este modo el resultado de la búsqueda sería:

\begin{tabular}{|c|c|}
\hline \multicolumn{2}{|l|}{ 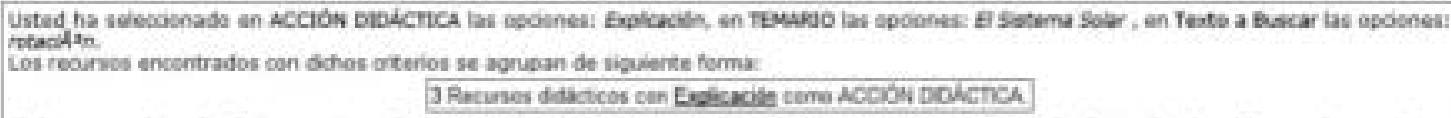 } \\
\hline 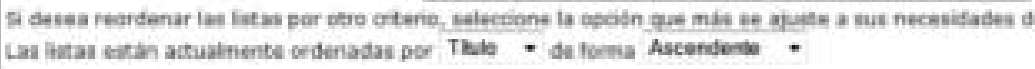 & Dulue antena \\
\hline \multicolumn{2}{|l|}{ Explicación - Pagina 1 - } \\
\hline \multicolumn{2}{|c|}{ 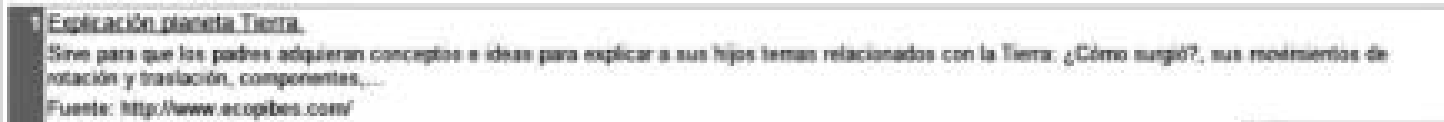 } \\
\hline Facta $17 / 2$ asot & \\
\hline \multicolumn{2}{|l|}{ 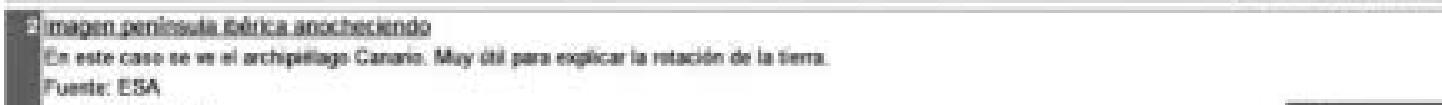 } \\
\hline Fecha tartwoset & 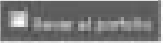 \\
\hline \multicolumn{2}{|c|}{ 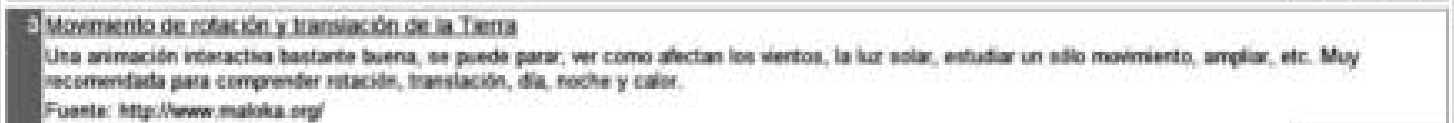 } \\
\hline & \\
\hline
\end{tabular}

Se puede observar que ha dado con tres recursos útiles que nos pueden servir de manera específica para explicar el concepto de rotación de los planetas.

Este tipo de búsqueda es el que más se ha utilizado por los miembros de la red social, y el más útil para usuarios esporádicos del proyecto MARIA.

\section{Preparar un plan de actividades con recursos DE LA RED SOCIAL Y EXTERNOS. El PORFOLIO}

Hemos descrito dos ejemplos de búsqueda, el primero de ellos estaría orientado hacia $r$ profesionales de la educación, mientras que el segundo sería más utilizado por madres y padres que necesitaran una ayuda puntual para realizar alguna actividad formativa concreta.

Sin embargo, la red social también dispone de un recurso muy importante que sirve para planificar un conjunto de actividades a una determinada persona y compartir esa experiencia no sólo con la red social, sino con el "resto del mundo".

Sea cual sea el tipo de búsqueda realizada, el centro de recursos de la red social muestra el conjunto de recursos útiles, clasificados por actividades. Sin embargo, el usuario puede agruparlos como desee, modificarlos e incluso añadir recursos propios.

Todos los recursos que el sistema muestra tienen en el lado derecho una casilla denominada "llevar al porfolio". 


\section{Tablas de mutiokar}

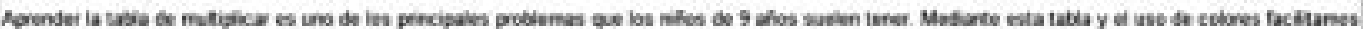

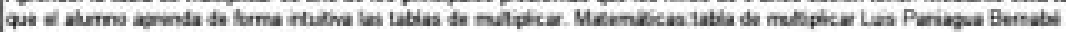

Fierte tha Mwwwiachicon con

Fecha tortaratos

Arbiacemutiolicar

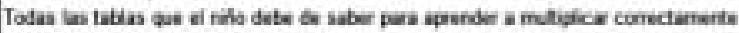

Fechas teresonos

\begin{tabular}{|c|c|}
\hline \multicolumn{2}{|l|}{ Exercioo - Página 1 - } \\
\hline 1 jasrense matematcal en & \\
\hline 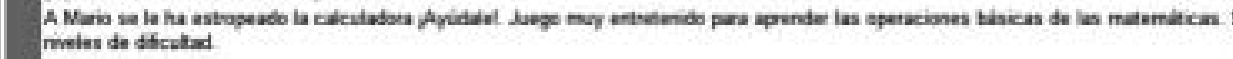 & Se utecent vation \\
\hline Pecha Tupseont & 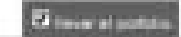 \\
\hline
\end{tabular}

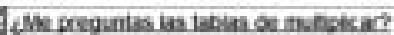

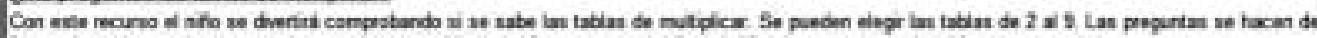

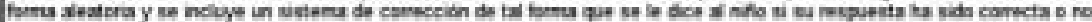

\section{Jatidad menta}

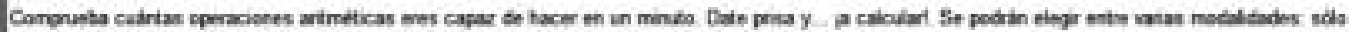

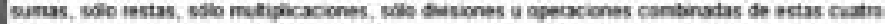

\section{A corenge a manctica}

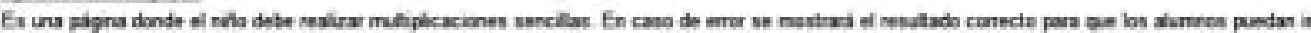

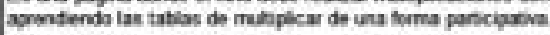

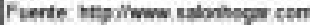

recha Det2aroos

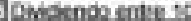

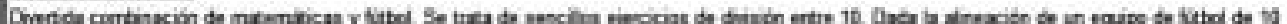

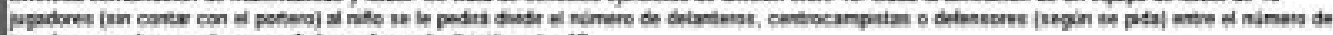

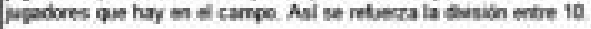

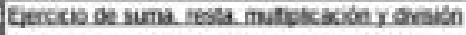

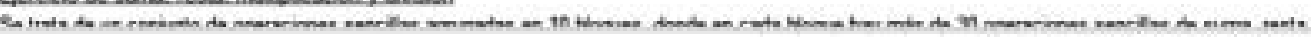

Observe que se han "marcado" varios recursos en la parte derecha de la imagen.

El usuario puede marcar cualquiera de esas casillas y posteriormente pulsar el botón "Generar porfolio".
El resultado es un documento "tipo Word" editable desde Internet, dicho documento lo podremos modificar. El resultado para el ejemplo sería el siguiente. 
ACTIVIDADES PARA REALIZAR EL PRIMER DÍA

phis

Twis atrackia

Deseripenon

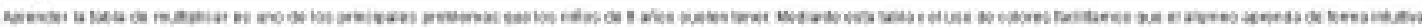

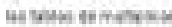

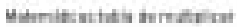

Lesponaziaterat

Drestitn wes

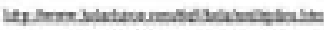

Thoulo

Twien turngetst

Descripeion

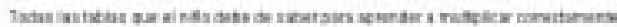

pereseiten wes

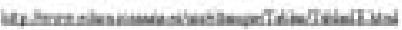

\section{ACTIVIDADES PARA REALIZAR EL SEGUNDO DÍA}

mise

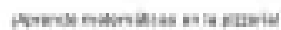

Descripioton

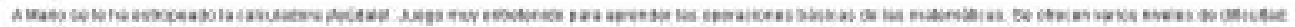

picessian Wob

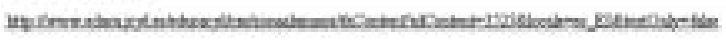

Titulo

Nabuenum

Deseripeion

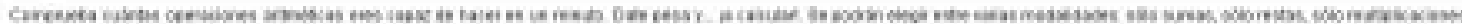

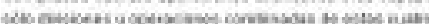

Direscian Wob

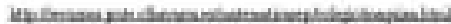

ACTIVIDAD PARA REALIZAR EL TERCER DÍA

Thula

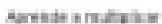

Deserpeten

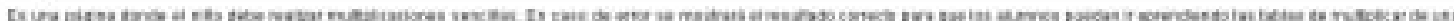
trmantacentre

bressien web

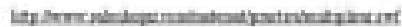

La planificación específica dividida en tres bloques, señalizando qué actividades hay que realizar cada día es una composición propia, no esta generada por el sistema. Se trata de un Porfolio dividido en función de una planificación acordada previamente 
Observe que el sistema ha generado un documento sobre el cual (letras en mayúsculas y en rojo) se ha añadido texto. El resultado podría ser un conjunto de recursos con una planificación para atender un caso de diversidad concreto.

Si este conjunto de recursos funciona, el profesor puede compartir bien a través de la propia red o bien por otros medios (en un foro, wiki, correo electrónico, etc.) ese conjunto de recursos. Si a él le ha funcionado seguramente a otras personas que tengan que atender la misma diversidad también les funcionará.

\section{Conclusiones}

Los miembros de la red social comenzaron cuando sus hijos tenían 8 años y se ha permanecido en ella hasta que han cumplido los 12; es decir, los miembros de la red social han cooperado de forma invisible durante tres cursos $\left(4 .^{\circ}, 5{ }^{\circ} \mathrm{y}\right.$ 6. de primaria); nadie sabía quién había aportado los recursos (únicamente se sabía que eran personas del mismo perfil y necesidad). Desde el punto de vista del individuo que conforma la red social la aportación de recursos se ha mantenido estable, no obstante como a medida que avanzaba el tiempo se apuntaban más personas a la red social, la cooperación fue aumentando.

Actualmente, de las personas que iniciaron la red social quedan muy pocas que participen; en su lugar han ido comunicando a familiares y amigos la posibilidad de parti- cipar en la red. Sin embargo, la red social continúa ya que siempre hay personas que necesitan recursos para ayudar a los niños con las tares formativas.

Las redes sociales orientadas a las personas están teniendo un gran éxito a nivel mundial. Sus miembros se cuentan por millones y las visitas se corresponden a información relacionada con las personas que conforman esas redes sociales.

Las redes sociales orientadas a los recursos también se han consolidado a nivel mundial. Las redes que organizan sus recursos con un fin determinado, como el expuesto en este artículo, están en un estado incipiente, sin embargo, su eficacia para la mejora de la competitividad personal y corporativa es más directa que el resto de las redes. Si además se integra con las anteriores estaremos introduciendo las redes sociales en las propias organizaciones.

Uno de los problemas que tienen las redes sociales orientadas a los recursos es su crecimiento, a medida que crece el número de recursos, su búsqueda se hace más complicada. Por tanto, es necesario disponer de herramientas tecnológicas que permitan que la eficacia de la red (facilidad para encontrar el recurso más adecuado) sea mayor cuanto mayor número de recursos existan.

El centro de recursos que ha originado la red social del proyecto MARIA la pueden encontrar y utilizar en http:// www.proyectomaria.org/

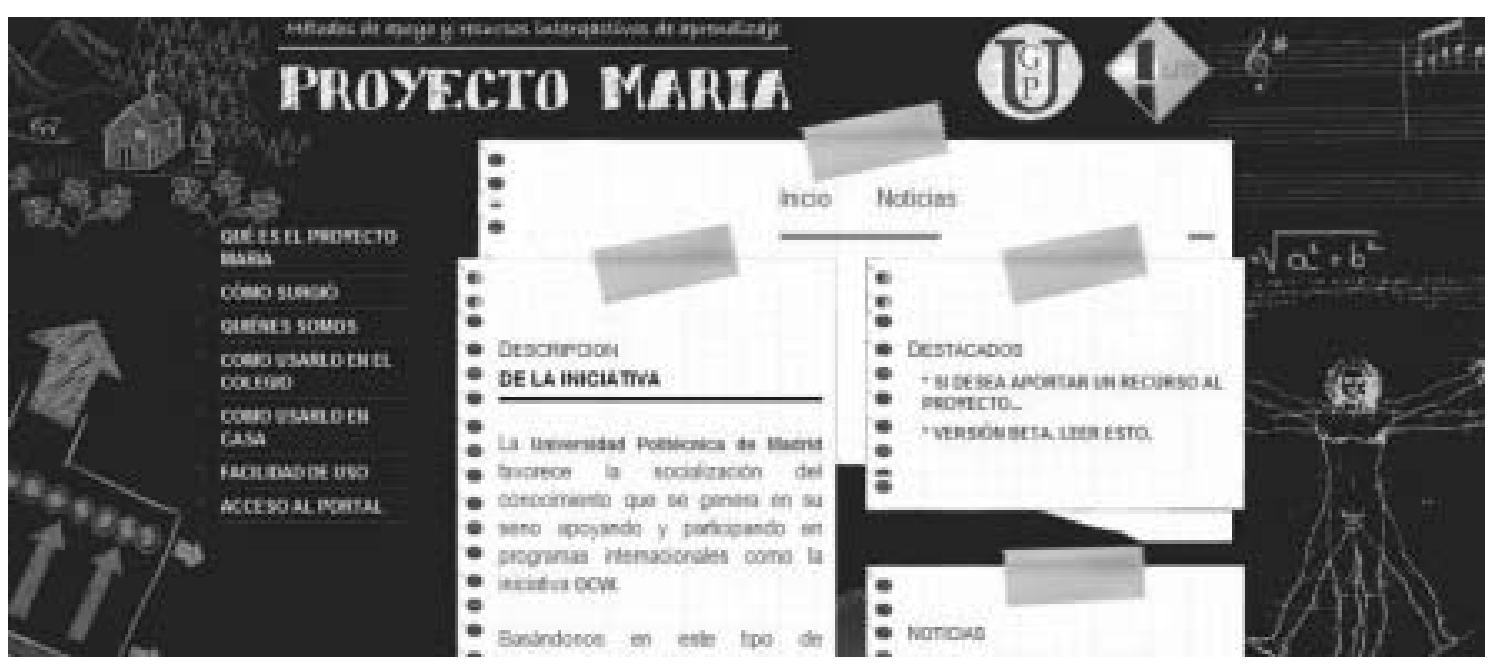




\section{NOTAS}

1 Laboratorio de Innovación en Tecnologías de la Información. Departamento de Matemática Aplicada y Métodos Informáticos. Universidad Politécnica de Madrid. http://www. liti.es/.

2 Visor del Ministerio de Medio Ambiente. http://sigpac.mapa.es/fega/ visor.

3 COCO. Clasificador y Organizador de Conocimiento por Ontologías. http:// www.centrorecursos.com/liti/website/ampliar.asp?d=680.

4 El COCO permite generar cualquier tipo de ontología fuerte. Una vez definida, el usuario de la red social no la podrá modificar, aunque sí los administradores de la misma. Las ontologías fuertes cambian en función de la red social; por ejemplo si la red social es para gestionar historias clínicas, tendrá una ontología fuerte distinta a la que tiene la red social MARIA.

\section{WEBGRAFÍA}

What is Web 2.0. Tim 0'Reilly.

http://www.oreillynet.com/pub/a/oreilly/ tim/news/2005/09/30/what-is-web-20. html

George Siemmens. Connectivism.

http://www.elearnspace.org/Articles/connectivism.htm

\section{BIBLIOGRAFÍA}

Nonaka, Ikujiro (2006): La organización creadora de conocimiento, 2006. Editorial Oxford University Press.

OCDE (2005): Main Science \& Technology Indicators, volumen 2005/2, OCDE, Paris.
Recibido: 16 de marzo de 2009

Aceptado: 16 de abril de 2009 